\title{
Transtornos alimentares de ordem comportamental e seus efeitos sobre a saúde bucal na adolescência
}

\section{Behavioral eating disorders and their effects on oral health in adolescence}

\author{
Jefferson TRAEBERT* \\ Emília Addison Machado MOREIRA**
}

\begin{abstract}
TRAEBERT, J.; MOREIRA, E. A. M. Transtornos alimentares de ordem comportamental e seus efeitos sobre a saúde bucal na adolescência. Pesqui Odontol Bras, v. 15, n. 4, p. 359-363, out./dez. 2001.

Anorexia nervosa é um distúrbio de ordem comportamental definido como inanição deliberada e auto-imposta seguida de busca constante de magreza e medo mórbido de engordar. Bulimia nervosa também é um distúrbio de comportamento caracterizada pela ingestão compulsiva de grande quantidade de alimentos, alternando-se com ações dirigidas a evitar ganho de peso, como por exemplo o vômito auto-induzido. A ocorrência destas doenças tem aumentado, embora não existam dados epidemiológicos de ordem populacional no Brasil. Tais distúrbios geram freqüentemente erosão dental devido ao ambiente bucal extremamente ácido resultante do comportamento dos pacientes. O cirurgião-dentista pode ser o primeiro profissional a detectar estas doenças. Assim, o objetivo deste trabalho é contribuir para a familiarização do cirurgião-dentista com os primeiros sinais e sintomas destas importantes doenças de ordem comportamental.
\end{abstract}

UNITERMOS: Anorexia nervosa; Bulimia nervosa; Saúde bucal; Erosão de dente; Adolescência.

\section{INTRODUÇÃO}

A adolescência começa com a puberdade, fase em que ocorrem mudanças morfológicas e psicológicas que se aproximam da condição de adulto. São considerados adolescentes, segundo a Organização Mundial de Saúde, os indivíduos entre 10 e 19 anos de idade $^{17}$.

Alguns aspectos do comportamento do adolescente podem ter implicações no crescimento e na saúde. Os extremos de consumo alimentar podem caracterizar-se em desordens alimentares, incluindo anorexia nervosa, bulimia nervosa e/ou a obesidade, podendo ser refletido no crescimento somático, composição corporal e função menstrual ${ }^{9}$.

A anorexia nervosa é um distúrbio de comportamento conceituado como inanição deliberada e auto-imposta, seguida de uma implacável busca de magreza e por um medo mórbido de engordar, levando a sérios graus de emagrecimento ${ }^{4}$. A anemia normocítica, normocrômica, assim como a leucopenia e trombocitopenia são comuns na anorexia nervosa. Tais condições retornam à normalidade com a normalização da alimentação ${ }^{16}$.

Também classificada como distúrbio de comportamento, a bulimia nervosa é caraterizada por ingestão compulsiva e rápida de grande quantidade de alimentos com pouco ou nenhum prazer, alternando-se comportamentos dirigidos a evitar o ganho de peso como por exemplo, vomitar, usar excessivamente laxantes e diuréticos e submeter-se a períodos de restrição alimentar severa. Tais comportamentos também se devem ao medo mórbido de engordar ${ }^{1}$.

A prevalência da anorexia e bulimia nervosas têm aumentado nos últimos anos ${ }^{13}$, não existindo dados estatísticos para o Brasil ${ }^{1}$. Na Inglaterra, a prevalência da anorexia nervosa varia entre $0,1 \mathrm{e}$ $1 \%$ entre jovens do sexo feminino, com 4.000 novos casos por ano ${ }^{7}$. A idade média de ocorrência é aos 16 anos, com uma maior prevalência entre 12

\footnotetext{
*Doutorando em Odontologia, Área de Concentração Odontopediatria - Universidade Federal de Santa Catarina. Professor do Curso de Odontologia da Universidade do Sul de Santa Catarina.

**Doutora em Ciência dos Alimentos. Professora Titular do Departamento de Nutrição e do Programa de Pós-Graduação em Odontologia da Universidade Federal de Santa Catarina.
} 
TRAEBERT, J.; MOREIRA, E. A. M. Transtornos alimentares de ordem comportamental e seus efeitos sobre a saúde bucal na adolescência. Pesqui Odontol Bras, v. 15, n. 4, p. 359-363, out./dez. 2001.

e 13 anos. Cerca de 90 a 95\% dos casos são de mulheres brancas jovens, afluentes e com inteligência pelo menos normal ${ }^{14}$.

A bulimia nervosa é mais comum, com prevalência entre 1 e $2 \%$ entre meninas jovens, sendo que em relação ao sexo ocorre em uma proporção de 10:1 em favor do sexo feminino. A idade média de ocorrência é aos 25 anos $^{7}$. A incidência de desordens de alimentação, excetuando-se a obesidade, aumenta com a melhoria da condição socioeconômica ${ }^{14}$.

No que concerne ao peso corporal, na anorexia os doentes apresentam-se com cerca de $15 \%$ a menos do peso ideal enquanto na bulimia esta relação pode variar de $10 \%$ a menos do peso ideal até a obesidade $^{8}$.

No caso da anorexia nervosa, a doença inicia-se a partir de uma dieta restritiva e persistente, evitando-se alimentos que levam ao aumento de peso, como os carboidratos. Fatores estressantes para o paciente e/ou sua família, entre os quais, perdas, separações, mudanças etc. podem servir como desencadeantes da doença. O indivíduo passa a viver em função da dieta, do peso e da forma corporal. O curso da doença caracteriza-se por contínua e importante redução de peso. A distorção da imagem corporal existe com freqüência, mas não em todos os casos. Quando acontece tal distorção, a pessoa percebe-se gorda, mesmo apresentando estágios avançados de desnutrição. A ausência de inquietude com a doença é característica da anorexia nervosa pois os pacientes recusam-se a reconhecerem-se como doentes ${ }^{4}$. Considerando-se estes sintomas, pode-se avaliar a sua influência na saúde e no estado nutricional dos pacientes anoréxicos.

Os hábitos alimentares tornam-se secretos, bizarros e ritualizados. Há episódios de ingestão exagerada de alimentos antes considerados proibidos. Segue-se a indução do vômito, uso de laxantes e diuréticos ou a prática excessiva de exercícios com a finalidade de neutralizar tal ingestão, sempre com o objetivo de alcançar a magreza pretendida. Tais atitudes resultam em isolamento social progressivo ${ }^{5}$.

Alterações no padrão familiar são relatados como fatores de desenvolvimento e manutenção do transtorno alimentar. Destacam-se a existência de alianças encobertas entre os membros da família, alternância de culpabilidade, falhas de resolução de conflitos, distorção na comunicação, confusão de papéis entre os membros da família, ausência de limites individuais e falta de senso de identidade pessoal ${ }^{12}$.

No que se refere à bulimia nervosa, os critérios de diagnóstico mais amplamente aceitos incluem episódios recorrentes de consumo alimentar compulsivo, os chamados episódios bulímicos que se caracterizam pelo ato de comer repetidas vezes em pequenos intervalos. Há sensação de perda de controle sobre a própria alimentação, comportamentos recorrentes e inapropriados de compensação para prevenir o ganho de peso, como vômito auto-induzido, abuso de laxantes e diuréticos, jejum ou dieta restrita e exercícios vigorosos. Os episódios bulímicos e comportamentos compensatórios ocorrem em média duas vezes por semana durante pelo menos três meses e ocorre uma auto-avaliação indevidamente influenciada pela forma e pesos corporais. A primeira característica clínica é dada pela descrição de que o paciente ingere compulsivamente, com perda de controle, grande quantidade de alimento em um curto espaço de tempo, ou às vezes em um longo ritual de várias horas ou uma noite inteira. Normalmente, tais episódios ocorrem às escondidas, e são revestidos de vergonha, sensação de culpa e desejo de autopunição ${ }^{15}$.

Tanto na anorexia nervosa, em que o comportamento bulímico vem sendo descrito em $25 \%$ dos casos, quanto na bulimia nervosa, o ato de vomitar para controlar peso proporciona problemas dentais e o cirurgião-dentista é potencialmente o primeiro profissional da Saúde a diagnosticar a doença, devido às perdas de substância dental resultantes. Tais perdas de substância são chamadas de erosão dental ou perimólise e são causadas pela regurgitação de ácido gástrico e vômito ${ }^{2,12}$.

Assim sendo, o objetivo deste artigo foi revisar a literatura sobre a relação entre anorexia e bulimia nervosas e a saúde bucal, descrever os sinais e sintomas, contribuindo para que o cirurgião-dentista possa detectá-los precocemente, oferecer tratamento odontológico adequado e referir o paciente para assistência especializada.

\section{RELAÇÕES COM A SAÚDE BUCAL}

Como o cirurgião-dentista pode ser o primeiro profissional da Saúde a suspeitar da anorexia e bulimia nervosas ${ }^{2,12}$, devido aos sinais e sintomas de erosão dental resultantes de um ambiente bucal cronicamente ácido ${ }^{16}$, há a necessidade de que esteja preparado para um manejo adequado do paciente. O objetivo inicial é a obtenção de sua con- 
TRAEBERT, J.; MOREIRA, E. A. M. Transtornos alimentares de ordem comportamental e seus efeitos sobre a saúde bucal na adolescência. Pesqui Odontol Bras, v. 15, n. 4, p. 359-363, out./dez. 2001.

fiança, gerando conseqüentemente um melhor resultado no tratamento odontológico e possibilitando sua referência para serviços com abordagem multidisciplinar incluindo profissionais psicoterapeutas, médicos e nutricionistas, além do próprio cirurgião-dentista ${ }^{6}$.

$\mathrm{Na}$ anamnese, é importante que o cirurgiãodentista tenha a habilidade de abordar o assunto de forma que se obtenha a confiança do paciente, inquirindo sobre os hábitos alimentares e a possibilidade de existência de problemas gastrointestinais, ao invés de dirigir perguntas diretas acerca da anorexia e bulimia nervosas ${ }^{2}$. Deve-se considerar que esta postura pode, de certa forma, levar a resultados positivos do ponto de vista do tratamento do transtorno alimentar, pois possibilita a melhora de sua auto-estima por meio do tratamento odontológico do paciente ao longo do tempo, evitando a deterioração dos dentes ${ }^{12}$.

Para ROBB; SMITH ${ }^{10}$ (1996), os critérios de diagnóstico para erosão dental resultante de episódios bulímicos ou de vômito por anorexia caracterizam-se por erosão severa nas faces palatais dos dentes anteriores superiores (Figura 1); erosão moderada nas faces vestibulares destes mesmos dentes; faces linguais dos dentes anteriores inferiores e posteriores não afetadas; erosão com aspecto semelhante às das faces palatais dos dentes anteriores, nos dentes posteriores superiores; erosão variável nas faces oclusais e vestibulares dos rações com aspecto de ilhas e ausência de manchas nas superficies com erosão.

HAZELTON; FAINE ${ }^{6}$ (1996) relatam que um dos principais problemas para o dentista no trabalho com pacientes com desordens alimentares advém dentes posteriores superiores e inferiores; restau-

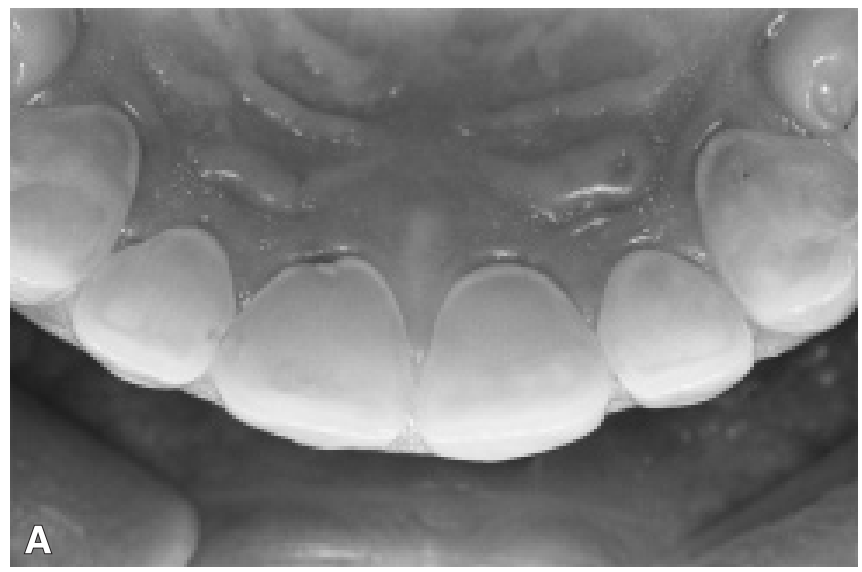

de seus problemas psicológicos. Normalmente, eles são bastante compulsivos em seus comportamentos diários. Sua higiene bucal é bastante meticulosa, repetitiva, e muitas vezes, seguida de escovações agressivas após o ato de vomitar, podendo resultar em severa abrasão. Nestes casos, o cirurgião-dentista deve alertar o paciente sobre o perigo destas escovações excessivas após o vômito e prescrever bochechos com água e bicarbonato de sódio para neutralizar o $\mathrm{pH}$ do ambiente bucal ${ }^{2,13}$.

BURKE et al. ${ }^{2}$ (1996) relatam que o manejo do paciente anoréxico/bulímico em relação à saúde bucal deve envolver cuidados emergenciais, educação do paciente, cuidado pré-restaurador (ou adequação de meio), restaurações e manutenção.

Dentre os cuidados emergenciais, encontramse a procura pelo alívio da dor e melhora da estética. Como alívio da dor, propõe-se a proteção da dentina indevidamente exposta com a utilização de cimentos de ionômero de vidro, vernizes fluoretados ou, se necessário, tratamento endodônti $\mathrm{co}^{2}$.

É fundamental uma abordagem de educação alimentar e saúde bucal concomitante ao tratamento restaurador, principalmente entre pacientes com má higiene bucal e ataques cariogênicos severos devido à ingestão de grandes quantidades de carboidratos. Em pacientes sem esses problemas, a educação em saúde deve enfocar o aumento de conhecimento sobre sua situação e os riscos dela decorrentes. Deve-se prescrever a utilização de cremes dentais com alta concentração de flúor e com baixa abrasividade, além da utilização de bochechos com substâncias neutralizadoras da acidez, como bicarbonato de sódio. Soluções neutras

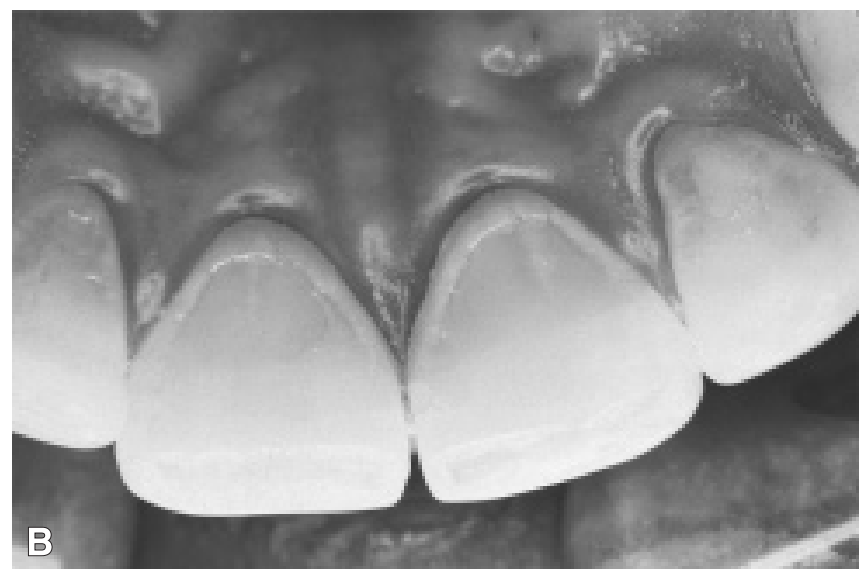

FIGURA 1 - A e B: Acentuado desgaste de esmalte e dentina nos dentes anteriores superiores; perda de estrutura de aspecto uniforme; tecido gengival saudável. 
TRAEBERT, J.; MOREIRA, E. A. M. Transtornos alimentares de ordem comportamental e seus efeitos sobre a saúde bucal na adolescência. Pesqui Odontol Bras, v. 15, n. 4, p. 359-363, out./dez. 2001.

de flúor a 0,05\% também são úteis na redução da erosão e da sensibilidade subseqüente ${ }^{2}$.

$\mathrm{O}$ aconselhamento dietético no sentido de evitar alimentos e bebidas ácidas, reduzindo a freqüência de sua ingestão, principalmente como última ingestão noturna, tem sido relatado como importante contribuição no tratamento dos pacientes acometidos pelas doenças ${ }^{13}$.

O tratamento restaurador deve ser realizado como uma forma de auxiliar o tratamento psicológico, na tentativa de aumentar a auto-estima do paciente ${ }^{2}$. Tal tratamento pode variar desde uma simples restauração até reabilitações bucais complexas em pacientes com perda de dimensão vertical. O tratamento deve ter como objetivo cobrir tecido dentinário exposto para evitar perdas ainda maiores de tecido dental ${ }^{2}$.

MILOSEVIC $^{8}$ (1999) relata que as restaurações de compósitos não são solúveis em ácido, e são preferiveis às restaurações de cimento de ionômero de vidro. A natureza conservadora das restaurações à base de adesivos dentinários facilitam a restauração de dentes de pacientes bulímicos.

Para HAZELTON; FAINE ${ }^{6}$ (1996), o planejamento do tratamento restaurador para estes pacientes é um desafio para o dentista. Se houver perda extensa de substância dental, o plano de tratamento normalmente deverá contemplar uma reabilitação bucal completa com restaurações bastante complexas. Mesmo com o intuito de melhorar a estética e de ser bastante cooperativo com as medidas preventivas prescritas, o paciente normalmente continua com seu hábito de vômito auto-induzido. Isto aumenta o risco de insucesso das restaurações pelo fato de as estruturas dentais de suporte permanecerem em um ambiente extremamente ácido. Assim, também restaurações provisórias tendem a sofrer erosão muito facilmente se tal hábito permanecer.

BURKE $^{3}$ (1998) relata a necessidade do desenvolvimento de técnicas apropriadas para tratar satisfatoriamente a perda severa de substância dental que pode ocorrer em pacientes bulímicos. Acredita que técnicas que requeiram mínima intervenção são mais apropriadas pois tais pacientes podem sofrer mais com a má aparência dental proveniente da perda de substância dental. A utilização de coroas de cerâmica pura, cimentadas sobre a dentina remanescente, com material adesivo é relatado como uma boa alternativa nestes casos. Tais técnicas requerem pouco tempo de tratamento e podem de forma rápida ajudar a elevar a auto-estima dos indivíduos doentes, decorrentes da insatisfação com sua auto-imagem.
RYTÖMAA et al. $^{11}$ (1998) relatam que nem todos os bulimicos apresentam erosão dental e que os fatores associados com a ocorrência e a severidade da condição são o tempo de duração da doença, a freqüência dos episódios de vômito e a quantidade de saliva. A saliva reduz a acidez. Portanto, em pacientes com fluxo salivar baixo, a acidez permanece, principalmente no dorso da língua, razão pela qual as faces palatinas dos dentes anteriores são mais afetadas. Os autores relatam também desgastes dos dentes, principalmente relacionados à mastigação e à escovação vigorosa em pacientes bulímicos, após o episódio de vômito. Neste estudo, os pacientes bulímicos tiveram uma maior prevalência de cárie em superficies proximais e palatinas do que pacientes controles, mas não houve diferença significativa nos hábitos de higiene e no estado periodontal.

HAZELTON; FAINE ${ }^{6}$ (1996) relatam que a atividade de cárie parece ser similar à da população em geral, entretanto em pacientes com lesões de cárie ativas, a velocidade na qual novas lesões se desenvolvem dificulta demasiadamente o tratamento. $\mathrm{O}$ periodonto pode estar afetado nas papilas que se mostram aumentadas devido à irritação constante advinda do vômito ácido. A mucosa bucal também sofre os efeitos de tal comportamento. Importante notar que o periodonto e a mucosa bucal podem estar afetados devido à medicação que os pacientes possam estar utilizando, como os anticolinérgicos, cujo uso prolongado induz à xerostomia e aumento da papila gengival.

Além da erosão, outras manifestações das desordens de alimentação podem incluir aumento assintomático das parótidas, xerostomia, irritações da mucosa oral, queilite, sensibilidade dental à alteração de temperatura, mordida aberta anterior e cáries radiculares ${ }^{11}$.

Assim, sugere-se que o cirurgião-dentista pode desempenhar um papel fundamental no diagnóstico de distúrbios de alimentação de ordem comportamental, devido à presença da erosão dental. Para isso, há a necessidade de o profissional estar familiarizado com os sinais das doenças e preparado para tratar e encaminhar os pacientes que apresentem tais distúrbios, contribuindo para o tratamento do transtorno alimentar.

\section{AGRADECIMENTOS}

Ao Professor Doutor Antônio Carlos Cardoso e à Professora Doutora Liliane Janete Grando, da Universidade Federal de Santa Catarina, por cederem as fotografias constantes neste artigo. 
TRAEBERT, J.; MOREIRA, E. A. M. Transtornos alimentares de ordem comportamental e seus efeitos sobre a saúde bucal na adolescência. Pesqui Odontol Bras, v. 15, n. 4, p. 359-363, out./dez. 2001.

TRAEBERT, J.; MOREIRA, E. A. M. Behavioral eating disorders and their effects on oral health in adolescence. Pesqui Odontol Bras, v. 15, n. 4, p. 359-363, out./dez. 2001.

Anorexia nervosa and bulimia nervosa are serious eating disorders that affect a significant number of adolescents and young adults. Individuals with anorexia nervosa tend to ignore or deny their excessive dieting and may present purging habits. The individual with bulimia nervosa spends great effort and time in compensating the effects of binge eating with dieting and fasting, self-inducing vomiting, utilization of laxatives or overexercising. The incidence of those behavioral disorders seems to be increasing, but there are no epidemiological data on that matter in Brazil. The dental practitioner has an important role in identifying these disorders since toothwear is very frequent due to the acidic oral environment caused by vomiting. The dentist can help to minimize the effects of anorexia and bulimia on the dentition. The objective of this study is to review the features of those behavioral eating disorders and to highlight the importance of being prepared to diagnose them and implement a comprehensive treatment of patients.

UNITERMS: Anorexia nervosa; Bulimia nervosa; Oral health; Tooth erosion; Adolescence.

\section{REFERÊNCIAS BIBLIOGRÁFICAS}

1. AZEVEDO, A. M. C.; ABUCHAIM, A. L. G. Bulimia nervosa: classificação diagnóstica e quadro clínico. In: NUNES, M. A. A. et al. Transtornos alimentares e obesidade. Porto Alegre : Artmed, 1998. p. 31-39.

2. BURKE, F. J. T.; BELL, T. J.; ISMAIL, N.; HARTLEY, P. Bulimia: implications for the practicing dentist. Brit Dent J, v. 180, n. 11, p. 421- 426, Jun. 1996.

3. BURKE, F. J. Treatment of loss of tooth substance using dentine-bonded crowns: relate of a case. Dent Update, v. 25, n. 6, p. 234-240, July/Aug. 1998.

4. CASTRO, J. M.; GOLDSTEIN, S. J. Eating attitudes and behaviors of pre- and postpuberal females: clues to the etiology of eating disorders. Physiol Behav, v. 58, n. 1, p. 15-23, Jan. 1995.

5. GIDWANI, G. P.; ROME, E. S. Eating disorders. Clin Obst Gyn, v. 40, n. 3, p. 601-615, Sept.1997.

6. HAZELTON, L. R.; FAINE, M. P. Diagnosis and dental management of eating disorder patients. Int $\mathbf{J}$ Prosthodont, v. 9, n. 1, p. 65-73, Jan. 1996.

7. HUGO, P. J.; LACEY, J. H. Eating disorders - diagnosis and management. Prim Care Psych, v. 2, n. 1, p. 87-100, Jan. 1996

8. MILOSEVIC, A. Eating disorders and the dentist. Brit Dent J, v. 186, n. 3, p. 109- 113, Feb. 1999.

9. PASTORE, D. R.; FISHER, M.; FRIEDMAN, S. B. Abnormalities in weight status, eating attitudes, and eating be- haviors among urban high school students. J Adolesc Health, v. 18, n. 1, p. 312-319, Jan. 1996.

10. ROBB, N. D.; SMITH, B. G. Anorexia and bulimia nervosa (the eating disorders): conditions of interest to the dental practitioner. J Dent, v. 24, n. 1-2, p. 7-16, Jan./Mar. 1996.

11. RYTÖMAA, I.; JÄRVINEN, V.; KANERVA, R.; HEINONEN, O. P. Bulimia and tooth erosion. Acta Odontol Scand, v. 56 , n. 1, p. 36-40, Feb. 1998.

12. SCHMIDT, U.; TREASURE, J. Eating disorders and the dental practitioner. Eur J Prosthodont Restor Dent, v. 5, n. 4, p. 161-167, Dec. 1997.

13. SHAW, L.; SMITH, A. J. Dental erosion - the problem and some practical solutions. Brit Dent J, v. 186, n. 3, p. 115-118, Feb. 1998.

14. WALDMAN, H. B. Is your next young patient pre-anorexic or pre-bulimic? ASCD J Dent Child, v. 65, n. 1, p. 52-56, Jan./Feb. 1998.

15. WALSH, B. T.; DEVLIN, M. J. Eating disorders: progress and problems. Science, v. 280, p. 1387-1390, May 1998.

16. WISEMAN, C. V.; HARRIS, W. A.; HALMI, K. A. Eating disorders. Med Clin North Am, v. 82, n. 1, p. 145-157, Jan. 1998.

17. WORLD HEALTH ORGANIZATION. Physical status: the use and interpretation of anthropometry. Report of a WHO expert committe. WHO technical report series, n. 854. Geneva, 1995, 452 p.

Recebido para publicação em 14/07/00

Enviado para reformulação em 15/05/01

Aceito para publicação em 25/06/01 